\title{
Comparação de custos entre sistema atual de transporte terceirizado e implantação de transportadora interna em indústria química no noroeste do Paraná
}

\author{
Cost comparison between the current system of third-party transportation and
} deployment of domestic carrier in chemical industry in northwestern Paraná

\author{
Luciane Hermes de Alencar $^{1 *}$; Edson Pereira da Mota ${ }^{2}$
}

\author{
${ }^{1}$ Raudi Indústria e Comércio Ltda - Engenheira Química - Rua Ana Nery, 135 - Centro - CEP 87780-000 \\ - Paraiso do Norte (PR), Brasil \\ 2 Universidade de São Paulo - Escola Superior de Agricultura "Luiz de Queiroz", PECEGE - Mestre em \\ Ciências - Avenida Pádua Dias, 11 - São Dimas - CEP 13418-900 - Piracicaba (SP), Brasil
}

\section{Resumo}

Com a competitividade em alta, cada vez mais as margens são estreitas e as expectativas dos clientes maiores. As empresas buscam reduzir custos para superar estes desafios e a Logística serve como peça chave neste processo. Diante disto, este trabalho tem como objetivo analisar a viabilidade da implantação de uma transportadora interna comparando com o sistema atual que é terceirizado. Afim de aumentar a margem de lucro sem aumentar o preço final para o cliente se tornando desta forma mais competitiva. $O$ trabalho foi realizado através de um levantamento de custo da opção atual terceirizada e os custos de ter uma transportadora interna. E por último a comparação entre esses custos, utilizando o método de comparação orçamentária, os resultados encontrados mostram que o projeto é viável do ponto de vista financeiro, pois traz retorno, no entanto quando dividimos esse retorno por kg de produto transportado fica evidente que é um valor que pouco agrega para a empresa, pois é menor que um centavo, e é este valor que torna o projeto inaceitável, pois substituir $100 \%$ da transportadora terceirizada traz riscos como desabastecimentos, atrasos e só seria viável assumir estes riscos se o valor agregado fosse no mínimo 10 vezes maior.

Palavras-chave: logística, orçamentária, tercerização

\section{Abstract}

With high competitiveness in increasingly narrow margins are higher and the expectations of customers. Companies seek to reduce costs to overcome these challenges and Logistics serves as a key element in this process. Given this, this paper aims to examine the feasibility of installing an internal carrier compared to the current system that is outsourced. In order to increase the profit margin without increasing the final price to the customer becoming more competitive in this way. The study was conducted through a survey of current cost of outsourced option and the cost of having an internal carrier. Finally the comparison between these costs, using the method of budget comparison, our results show that the project is feasible from a financial standpoint, it brings back, however when we divide this return per $\mathrm{kg}$ of product transported is evident that it is a value that adds little to the company because it is smaller than a dime, and it is this value that makes the project unacceptable because replacing $100 \%$ of outsourced carrier carries risks as shortages, delays and would only be feasible to take these risks if the aggregate value were at least 10 times greater.

\footnotetext{
1 Autor correspondente: <lucianehermes@yahoo.com.br>

Enviado: 02 jun. 2015

Aprovado: 30 jun. 2015
} 
Keywords: logistics, budget, outsourcing

\section{Introdução}

O principal objetivo de qualquer empreendimento é obter o maior retorno possível sobre o investimento, para cumprir essa meta, a logística se destaca como fator decisivo à garantia que produtos estejam no lugar certo, no tempo exato e na condição requerida, pelo menor custo possível.

Devido à inexistência do desenvolvimento de sistema de transporte e armazenamento em épocas mais antigas, o consumo de mercadorias se limitava a aquilo que se conseguia produzir por suas próprias mãos. As mercadorias mais necessárias não eram produzidas próximas aos lugares de consumo, por consequência, não estavam disponíveis à todos que a procuravam. Desta forma, os consumidores eram obrigados a viver perto do mercado produtor (Ballou, 2004).

Nesse contexto, os sistemas logísticos eficazes deram ao comércio condições de tirarem o máximo proveito da falta de uniformidade existente no mundo, ou seja, tirar proveito do que há de melhor em cada região. A essência do comércio é a logística, ela decisivamente contribui para que o padrão de vida econômico seja melhor sendo a ponte que faz a ligação entre locais de produção e mercados, separados por tempo e distâncias (Ballou, 2004). O fluxo de produtos segue em direção aos consumidores, o de informação parte dos consumidores até chegar ao alcance dos fornecedores (Bowersox; Closs, 2001).

Sem nenhum glamour gerencial e tradicionalmente vista como um conjunto de atividades, a logística começa a conquistar um novo espaço na agenda estratégica das empresas deixando, portanto, de ser relegada a níveis operacionais (Fleury; Lavalle, 1995).

Logística é o processo de planejamento, implantação e controle de fluxo eficiente e eficaz de mercadorias, serviços e das informações relativas desde o ponto de origem até o ponto de consumo com 0 propósito de atender às exigências dos clientes. (Ballou, 2004, p.27).

O sucesso ou o fracasso de qualquer negócio é determinado pelo nível de valor entregue ao cliente, as empresas bem sucedidas são aquelas que entregam mais valor ao cliente do que o seu concorrente (Christopher, 2007).

Para que as companhias possam competir e ganhar mercado, é necessário traçar uma estratégia que deixe sua cadeia de distribuição competitiva e, a terceirização, tem como um dos objetivos melhorar o desempenho dessa distribuição. 
$\mathrm{Na}$ iniciativa privada, o método da contratação de terceiros surgiu nos Estados Unidos antes da Segunda Guerra Mundial e, se consolidou como técnica de administração empresarial a partir da década de $50 \mathrm{com}$ o desenvolvimento acelerado da indústria (Leiria; Saratt, 1996).

No Brasil, a terceirização logística foi gradativamente implantada com a vinda das primeiras empresas multinacionais, principalmente as automobilísticas no início da década de 80. Essas fábricas adquiriam as peças de outras empresas, guardando para si a atividade fundamental de montagens de veículos (Queiroz, 1998).

Na logística, a redução de custos é a ideia básica da terceirização, ela também traz vantagem competitiva, uma vez que as empresas passam a priorizar a produção.

Para comprovar essa vantagem da terceirização, ou para substituí-la por alguma outra alternativa, é que foi realizado o estudo descrito neste artigo.

Por todos esses motivos, a busca por aumento de produtividade, a redução de custos fixos e novas oportunidades são incessantes e, uma das oportunidades visualizadas, para a indústria em estudo, é a implantação de uma transportadora interna, utilizando o sistema de agregação (motoristas com caminhões próprios).

A comparação realizada neste trabalho buscou esclarecer se a terceirização total, opção atual adotada pela empresa, é a alternativa mais rentável frente à implantação de transportadora interna. A fim de aumentar a margem de lucro sem aumentar o preço final para o cliente se tornando desta forma mais competitiva.

\section{Material e Métodos}

A empresa em estudo é uma indústria química de porte médio, situada no noroeste do Estado do Paraná. Seus produtos, apesar de não serem commodities, termo utilizado para designar bens para os quais existe uma procura sem atender à diferenciação de qualidade do produto no conjunto dos mercados, e entre vários fornecedores ou marcas, se comportam como tal, pois apresentam pouco valor agregado.

No Brasil existem três indústrias do mesmo segmento e há ainda a competição externa que tem maior ou menor influência em função do câmbio. O principal segmento atendido é o de nutrição animal, onde as margens são ainda menores, esse segmento responde por $60 \%$ do total.

O estudo foi realizado em 3 etapas. A primeira etapa consistiu do levantamento dos custos da transportadora terceirizada e sua margem de lucro bruta. A segunda etapa considerou o levantamento dos custos da transportadora interna e, a terceira e 
última etapa, realizou a comparação entre os custos envolvidos em ambas alternativas para a escolha da melhor.

Etapa 1: Levantamento dos custos da transportadora terceirizada

Para este estudo foi utilizado um método analítico que se baseia em estudos a "posteriori", ou seja, depende de experiência ou evidência empírica. Os métodos analíticos foram utilizados para estudar a situação atual, auxiliando na compreensão da realidade sobre a qual se deseja interferir (Hoffmann et. al., 1992).

Para levantar os custos de operação da transportadora terceirizada foi necessária a realização de pesquisa com os motoristas contratados pela mesma para a coleta de informações sobre os valores recebidos pelo transporte. Estes valores fazem parte das negociações diretas da transportadora terceirizada com o motorista, não sendo um valor tabelado, essa pesquisa foi realizada durante o carregamento dos mesmos, sempre por abordagem direta e individual. Os outros valores necessários foram valor pago a transportadora pela empresa, valor da carga e alíquota de ICMS. Estes foram retirados do conhecimento de transporte eletrônico, que é um documento emitido com o intuito de documentar, para fins fiscais, uma prestação de serviço de transporte de cargas realizada por qualquer modal (Rodoviário, Aéreo, Ferroviário, Aquaviário e Dutoviário). Sua validade jurídica é garantida pela assinatura digital do emitente (garantia de autoria e de integridade) e pela recepção e autorização de uso, pelo Fisco (Secretária da Fazenda, 2014).

Durante 0 mês de setembro foi realizada uma amostragem com aproximadamente $32 \%$ dos carregamentos, a escolha específica deste mês se deve, pois, historicamente setembro é o mês mais próximo da média anual de produtos expedidos. Esta amostragem cobre aproximadamente $70 \%$ dos clientes expedidos, pois na quantidade total, os clientes se repetem e, por consequência, os valores pagos para os fretes também já que dentro de um mês não há variação de custo para cargas enviadas a um mesmo destino.

A partir de todos os dados coletados foram elaborados os cálculos de custos da transportadora terceirizada. A equação 1 apresenta a Receita 1, obtida pela subtração do valor pago ao motorista pela transportadora do valor pago pela empresa para transportadora.

$$
\text { Receita 1= VPT - VPM }
$$

Onde, VPT: Valor pago pela empresa para transportadora; VPM: Valor pago ao motorista pela transportadora. 
O valor dos impostos foi calculado pela equação 2 , sempre sobre os valores pagos pela empresa à transportadora, por este ser o valor declarado no documento fiscal, conhecimento do transporte eletrônico.

Valor dos impostos $=$ VPT $-\left(\right.$ VPT $\left.^{\star}(100 \%-(I C M S+P I S / C O F I N S))\right)$

Onde, ICMS: alíquota que varia de acordo com o estado de destino (anexo A); PIS/COFINS: tributo no qual a empresa pode escolher o regime de tributação que se encaixa, a transportadora em questão se enquadra no sistema de lucro presumido, em termos gerais, trata-se de um lucro fixado a partir de percentuais padrões aplicados sobre a Receita Operacional Bruta - ROB.

Sobre o referido resultado somam-se as outras receitas auxiliares (receitas financeiras, aluguéis esporádicos, entre outras). Assim, por não se tratar do lucro contábil efetivo, mas uma mera aproximação fiscal, denomina-se de Lucro Presumido (Portal tributário, 2014). A soma dos dois impostos foi de 3,65\%.

Outro custo envolvido nesta etapa foi o seguro de mercadorias, que tem como propósito dar proteção a carga transportada contra danos ou perdas, visando apenas repor algum dano advindo da ocorrência de sinistro, e nunca proporcionar lucros com relação ao bem segurado. Através da equação 3 foi possível calcular o valor do seguro para cada carga, pois o valor do mesmo depende do valor da mercadoria transportada. Este foi multiplicado por uma taxa fixa, no caso da transportadora terceirizada o valor corresponde a $0,4 \%$, definido através de contrato com a seguradora.

$$
\text { Seguro }=\text { Valor da Carga }{ }^{*} 0,4 \%
$$

A Receita final, apresentada na equação 4, foi obtida subtraindo-se do valor da Receita 1 o valor dos impostos e o valor do seguro.

$$
\text { Receita final= Receita } 1-\text { Valor dos impostos }- \text { Seguro }
$$

A Receita final representa o valor obtido pela transportadora terceirizada em cada carga, dividindo-se o somatório dessas receitas pelo somatório dos valores pagos pela empresa para transportadora, tem-se a Margem obtida (Equação 5)

Margem Obtida $=($ Somatório Receita final/Somatório VPT)*100 
Com a margem obtida obteve-se a porcentagem de lucro que a transportadora obtém sobre os transportes realizados para a empresa e, para transformar essa porcentagem em valor, foi apurado o total de pagamentos realizados no mês de setembro. Desta forma, tem-se (Equação 6):

Lucro Mensal Transportadora $=$ Total faturado no mês * Margem obtida

O lucro mensal da transportadora terceirizada representa o valor para garantir os custos com a transportadora interna e, o projeto só será viável, se após a cobertura dos custos ainda obtiver lucro.

\section{Etapa 2: Custos da transportadora interna}

Para a implantação e abertura de uma transportadora, existe lei específica com as condições mínimas a serem atendidas, conforme segue:

Segundo a Lei no 11.442, de 5 de janeiro de 2007 para abertura de Empresa de Transporte Rodoviário de Cargas - ETC deverá: I - Ter sede no Brasil; II - Comprovar ser proprietária ou arrendatária de, pelo menos, 1 (um) veículo automotor de carga, registrado no País; III - Indicar e promover a substituição do Responsável Técnico, que deverá ter, pelo menos, 3 (três) anos de atividade ou ter sido aprovado em curso específico (BRASIL, 2007).

A empresa em estudo já cumpre com os dois primeiros itens da lei, os únicos custos adicionais da implantação da transportadora interna seriam relativos à mão de obra e administrativos.

Para levantar os custos com mão de obra, foram utilizados salários condizentes com a função na região, mais os encargos e benefícios oferecidos pela empresa para todos os colaboradores:

- Salário líquido: valor pago ao colaborador, deduzidos todos os impostos.

- Décimo terceiro salário: a gratificação de Natal foi instituída no Brasil pela Lei 4.090, de 13/07/1962, e garante que o trabalhador receba o correspondente a 1/12 (um doze avos) da remuneração por mês trabalhado. O seu valor, embora variável, foi aproximado ao de um salário mensal, podendo ser pago em uma ou mais prestações, de acordo com a legislação laboral de cada país.

- Férias: período de descanso anual, que deve ser concedido ao empregado após o exercício de suas atividades por um ano. Além do salário normal o colaborador tem direito a mais 1/3 do salário como abono. 
- FGTS: Fundo de Garantia por Tempo de Serviço, um depósito mensal, referente a percentual de $8 \%$ do salário e variáveis, se houver, do empregado, da qual o empregador deposita todo início de mês até o dia 07 na conta do FGTS do colaborador.

- INSS empresa, Terceiros: Contribuição mensal (e obrigatória) de encargo à Previdência Social da empresa, a incidência foi calculada pela Classificação Nacional de Atividades Econômicas - CNAE, onde se aplica a Tabela de Alíquotas por Fundo da Assistência e Previdência Social - FPAS.

- Acidente Trabalho: cálculo anual baseado no índice de acidentes da empresa, registrados com CAT, o cálculo encontra-se disponível no sítio do Ministério da Previdência Social - MPS, Fator Acidentário de Prevenção - FAP X o Riscos Ambientais do Trabalho - RAT.

- Refeição: Custo mensal da empresa com a refeição do colaborador, concedida sem nenhum custo para o mesmo.

- Vale Mercado: Valor concedido através do Cartão Vale Alimentação, cobre gastos com mercado.

- Transporte: Custo mensal da empresa com o transporte do colaborador dos pontos até a empresa e o retorno. Concedido sem nenhum custo para o mesmo.

Os custos administrativos foram baseados nos números reais de um mês e divididos pelo número de colaboradores do setor.

Se enquadram nestes custos as despesas com energia, água, internet, telefone, celular, assessoria jurídica, assessoria do programa, reembolsos de despesas para fins de trabalho externo e consultorias.

A soma desses dois valores (salário/encargos/benefícios e custos administrativos) resulta no custo adicional que a transportadora interna irá gerar.

\section{Etapa 3: Comparação entre os custos envolvidos}

Para esta etapa foi escolhido o método de comparação de grupo que permite planejar um sistema de operação melhor que o existente, trata-se de um método relativamente grosseiro, porém econômico, simples e de relativa rapidez de sua execução (Hoffmann et al., 1992).

Para essa comparação entre os grupos, utilizou-se o método dos orçamentos que tem como base o princípio que o plano que apresentar o melhor saldo positivo entre os custos e receitas será o adotado pela empresa. No entanto, dentro deste método, foi 
necessário levar em consideração aspectos de caráter não monetário, mas sociais, culturais, etc (Hoffmann et al., 1992).

$\mathrm{Na}$ última etapa foram comparados os valores encontrados nas duas primeiras etapas, a fim de verificar se a melhor opção financeira para a empresa é a manutenção da terceirização do transporte ou realizar a implantação de uma transportadora interna.

\section{Resultados e Discussão}

Etapa 1: Custos da transportadora terceirizada

Utilizando os dados amostrais obtidos da pesquisa de campo com os motoristas terceirizados (Anexo B) e a equação (1), tem-se o resultado da Receita 1, que foi o valor pago pela empresa em estudo à transportadora terceirizada - VPT, retirando-se o valor pago ao motorista pela transportadora terceirizada - VPM (Tabela 1). 
Tabela 1. Valor obtido para a Receita 1

\begin{tabular}{|c|c|c|}
\hline VPM & VPT & RECEITA 1 \\
\hline $\mathbf{R} \mathbf{\$}$ & $\mathbf{R} \mathbf{S}$ & $\mathbf{R} \$$ \\
\hline $1.400,00$ & $1.920,00$ & 520,00 \\
\hline $1.050,00$ & $1.470,00$ & 420,00 \\
\hline $3.000,00$ & $4.350,00$ & $1.350,00$ \\
\hline $2.850,00$ & $4.050,00$ & $1.200,00$ \\
\hline $1.400,00$ & $1.890,00$ & 490,00 \\
\hline $1.500,00$ & $2.025,00$ & 525,00 \\
\hline $1.300,00$ & $1.890,00$ & 590,00 \\
\hline $2.405,00$ & $3.330,00$ & 925,00 \\
\hline $2.200,00$ & $2.990,00$ & 790,00 \\
\hline $1.100,00$ & $1.560,00$ & 460,00 \\
\hline 800,00 & 945,00 & 145,00 \\
\hline $2.500,00$ & $3.150,00$ & 650,00 \\
\hline $2.400,00$ & $3.536,00$ & $1.136,00$ \\
\hline $2.600,00$ & $3.600,00$ & $1.000,00$ \\
\hline $1.000,00$ & $1.377,00$ & 377,00 \\
\hline $1.400,00$ & $1.600,00$ & 200,00 \\
\hline 800,00 & $1.080,00$ & 280,00 \\
\hline $2.900,00$ & $3.990,00$ & $1.090,00$ \\
\hline $1.000,00$ & $1.620,00$ & 620,00 \\
\hline $2.200,00$ & $3.000,00$ & 800,00 \\
\hline $1.100,00$ & $1.600,00$ & 500,00 \\
\hline $3.000,00$ & $4.350,00$ & $1.350,00$ \\
\hline $3.300,00$ & $4.590,00$ & $1.290,00$ \\
\hline $1.100,00$ & $1.404,00$ & 304,00 \\
\hline $1.400,00$ & $1.890,00$ & 490,00 \\
\hline $3.000,00$ & $4.800,00$ & $1.800,00$ \\
\hline $2.400,00$ & $3.600,00$ & $1.200,00$ \\
\hline $1.600,00$ & $1.890,00$ & 290,00 \\
\hline $1.000,00$ & $1.485,00$ & 485,00 \\
\hline $1.200,00$ & $1.957,50$ & 757,50 \\
\hline $2.500,00$ & $3.600,00$ & $1.100,00$ \\
\hline $2.200,00$ & $3.000,00$ & 800,00 \\
\hline $3.000,00$ & $4.160,00$ & $1.160,00$ \\
\hline 800,00 & $1.255,50$ & 455,50 \\
\hline 800,00 & 945,00 & 145,00 \\
\hline $2.400,00$ & $3.600,00$ & $1.200,00$ \\
\hline $2.200,00$ & $2.990,00$ & 790,00 \\
\hline $1.300,00$ & $1.822,50$ & 522,50 \\
\hline $2.600,00$ & $3.150,00$ & 550,00 \\
\hline $2.100,00$ & $3.536,00$ & $1.436,00$ \\
\hline $1.000,00$ & $1.282,50$ & 282,50 \\
\hline $1.100,00$ & $1.485,00$ & 385,00 \\
\hline $76.905,00$ & $107.766,00$ & $30.861,00$ \\
\hline
\end{tabular}

VPT: valor pago pela empresa em estudo à transportadora terceirizada; VPM: valor pago ao motorista pela transportadora terceirizada.

Este valor representa um resultado preliminar do lucro em relação aos dados amostrais. Para a transformação desse valor na margem sobre o total de produtos expedidos foi necessário calcular a margem bruta de lucro. 
Também foram obtidos os valores dos impostos, o valor da mercadoria, que reflete diretamente no valor do seguro contratado e, por fim, a receita final e a margem obtida.

Tabela 2. Margem obtida

\begin{tabular}{|c|c|c|c|c|}
\hline $\begin{array}{r}\text { VALOR DE } \\
\text { IMPOSTOS } \\
\end{array}$ & $\begin{array}{c}\text { VALOR DA } \\
\text { MERCADORIA }\end{array}$ & SEGURO & RECEITA FNAL & $\begin{array}{c}\text { MARGEM } \\
\text { OBTIDA }\end{array}$ \\
\hline $\mathbf{R} \$$ & $\mathbf{R} \$$ & $\mathbf{R} \$$ & $\mathbf{R} \mathbf{S}$ & $\%$ \\
\hline 70,08 & $35.801,60$ & 143,21 & 306,71 & 15,97 \\
\hline 156,56 & $16.130,44$ & 64,52 & 198,92 & 13,53 \\
\hline 680,78 & $35.400,00$ & 141,60 & 527,63 & 12,13 \\
\hline 633,83 & $45.000,00$ & 180,00 & 386,18 & 9,54 \\
\hline 295,79 & $18.630,00$ & 74,52 & 119,70 & 6,33 \\
\hline 316,91 & $16.605,00$ & 66,42 & 141,67 & 7,00 \\
\hline 295,79 & $19.170,00$ & 76,68 & 217,54 & 11,51 \\
\hline 521,15 & $42.550,00$ & 170,20 & 233,66 & 7,02 \\
\hline 467,94 & $31.500,00$ & 126,00 & 196,07 & 6,56 \\
\hline 56,94 & $26.000,00$ & 104,00 & 299,06 & 19,17 \\
\hline 100,64 & $15.120,00$ & 60,48 & $-16,12$ & $-1,71$ \\
\hline 492,98 & $30.975,00$ & 123,90 & 33,13 & 1,05 \\
\hline 553,38 & $37.280,00$ & 149,12 & 433,50 & 12,26 \\
\hline 563,40 & $41.700,00$ & 166,80 & 269,80 & 7,49 \\
\hline 215,50 & $18.630,00$ & 74,52 & 86,98 & 6,32 \\
\hline 250,40 & $15.727,50$ & 62,91 & $-113,31$ & $-7,08$ \\
\hline 39,42 & $15.930,00$ & 63,72 & 176,86 & 16,38 \\
\hline 424,94 & $42.300,00$ & 169,20 & 495,87 & 12,43 \\
\hline 253,53 & $16.065,00$ & 64,26 & 302,21 & 18,65 \\
\hline 469,50 & $29.700,00$ & 118,80 & 211,70 & 7,06 \\
\hline 250,40 & $13.050,00$ & 52,20 & 197,40 & 12,34 \\
\hline 680,78 & $35.400,00$ & 141,60 & 527,63 & 12,13 \\
\hline 488,84 & $19.440,00$ & 77,76 & 723,41 & 15,76 \\
\hline 149,53 & $16.537,50$ & 66,15 & 88,32 & 6,29 \\
\hline 295,79 & $18.630,00$ & 74,52 & 119,70 & 6,33 \\
\hline 751,20 & $36.000,00$ & 144,00 & 904,80 & 18,85 \\
\hline 563,40 & $36.000,00$ & 144,00 & 492,60 & 13,68 \\
\hline 201,29 & $16.200,00$ & 64,80 & 23,91 & 1,27 \\
\hline 54,20 & $18.285,00$ & 73,14 & 357,66 & 24,08 \\
\hline 306,35 & $14.175,00$ & 56,70 & 394,45 & 20,15 \\
\hline 563,40 & $39.300,00$ & 157,20 & 379,40 & 10,54 \\
\hline 469,50 & $34.500,00$ & 138,00 & 192,50 & 6,42 \\
\hline 651,04 & $39.190,00$ & 156,76 & 352,20 & 8,47 \\
\hline 45,83 & $15.930,00$ & 63,72 & 345,95 & 27,56 \\
\hline 100,64 & $15.120,00$ & 60,48 & $-16,12$ & $-1,71$ \\
\hline 563,40 & $41.700,00$ & 166,80 & 469,80 & 13,05 \\
\hline 467,94 & $31.500,00$ & 126,00 & 196,07 & 6,56 \\
\hline 285,22 & $17.955,00$ & 71,82 & 165,46 & 9,08 \\
\hline 492,98 & $34.650,00$ & 138,60 & $-81,57$ & $-2,59$ \\
\hline 553,38 & $37.280,00$ & 149,12 & 733,50 & 20,74 \\
\hline 46,81 & $15.255,00$ & 61,02 & 174,67 & 13,62 \\
\hline 232,40 & $17.415,00$ & 69,66 & 82,94 & 5,59 \\
\hline $15.073,72$ & $1.113 .727,04$ & 4.454,91 & $11.332,37$ & 10,52 \\
\hline
\end{tabular}


Após os cálculos realizados, chegou-se a margem de lucro da transportadora terceirizada sobre a empresa totalizando $10,52 \%$ do total pago a mesma. O total pago a transportadora no período considerado de estudo foi de $\mathrm{R} \$ 346.757,67$.

A obtenção destes valores permitiu calcular o lucro mensal obtido pela transportadora terceirizada (Equação 6).

Lucro Mensal Transportadora $=\mathrm{R} \$ 346.757,67 * 10,52 \%=\mathrm{R} \$ 36.478,91$

Este valor ainda não foi o lucro real da transportadora terceirizada, pois a mesma ainda necessita descontar os custos envolvidos com a mão-de-obra, mas como este é um custo comum às duas opções em estudo e, os gastos são particularidade da empresa terceirizada, este será o número utilizado na comparação para análise de comparação.

\section{Etapa 2: Custos de uma transportadora interna}

Conforme mencionado, dois custos principais foram levados em consideração para o levantamento dos custos de implantação e manutenção de uma transportadora interna, mão-de-obra e administrativos.

Para a operação, dois colaboradores foram suficientes. O colaborador 1 seria o responsável pelo operacional, contato com agenciadores, contratação dos motoristas, acerto dos valores, rastreamento de cargas, feedback para diretoria, indicadores de performance. O colaborador 2 seria o responsável pela parte administrativa, emissão dos conhecimentos de transporte, dados para contabilidade e financeiro.

Os custos envolvidos na contratação desses colaboradores estão apresentados na Tabela 3. 
Tabela 3. Custos mensais de mão-de-obra

\begin{tabular}{lclc}
\hline \multicolumn{2}{c}{ COLABORADOR 1 } & \multicolumn{2}{c}{ COLABORADOR 2 } \\
\hline \multirow{2}{*}{ SALARIO BRUTO } & $\mathbf{4 . 5 0 0 , 0 0}$ & SALARIO BRUTO & $\mathbf{2 . 5 0 0 , 0 0}$ \\
SALARIO LIQUIDO & $3.716,19$ & SALARIO LIQUIDO & $2.192,21$ \\
13o & 375,00 & 13 o & 208,33 \\
FÉRIAS & 375,00 & FÉRIAS & 208,33 \\
1/3 FÉRIAS & 125,00 & 1/3 FÉRIAS & 69,44 \\
FGTS & 60,00 & FGTS & 200,00 \\
INSS EMPRESA - 20\% & 900,00 & INSS EMPRESA - 20\% & 500,00 \\
TERCEIROS - 5,8\% & 261,00 & TERCEIROS - 5,8\% & 145,00 \\
ACID. TRAB. - 3,879\% & 174,56 & ACID. TRAB. - 3,879\% & 96,98 \\
REFEIÇÃO & 237,50 & REFEIÇÃO & $\mathbf{2 3 7 , 5 0}$ \\
VALE MERCADO & 206,64 & VALE MERCADO & $\mathbf{2 0 6 , 6 4}$ \\
TRANSPORTE & 147,06 & TRANSPORTE & 147,06 \\
\hline TOTAL & $\mathbf{6 . 8 7 7 , 9 5}$ & TOTAL & $\mathbf{4 . 2 1 1 , 5 0}$ \\
\hline
\end{tabular}

Assim tem-se que o a somatório dos custos totais mensais com mão-de-obra seriam de $\mathrm{R} \$ 11.089,45$. Considerando os custos administrativos (água, luz, telefone, internet, jurídico, consultoria do programa, etc.), estes representam total de $R \$ 5.200,00$ por colaborador.

Desta forma, para a implantação de uma transportadora interna seria necessário um custo mensal de $\mathbf{R} \$ \mathbf{2 1 . 4 8 9 , 4 5 .}$

Etapa 3: Comparação entre os as alternativas

A atual competição existente entre as empresas por seu espaço e sobrevivência no mercado, torna a busca por melhoria e eficiência em suas operações imprescindível para que a mesma possa obter vantagens competitivas. As pressões para a redução de custos e melhor atendimento a seus mercados consumidores, têm tornado a gestão de seus processos um desafio diário.

A alternativa de modificar o sistema logístico empregado pode atender a essa necessidade, dada tal justificativa foi realizada a comparação entre as duas alternativas propostas. 
Comparando os custos envolvidos com a transportadora terceirizada e interna, tem-se $R \$ 36.478,91-R \$ 21.489,45=R \$ 14.989,46$. Este resultado representa uma substituição de $100 \%$ no sistema, ou seja, a transportadora interna seria a única responsável pelo transporte. Se tiver uma substituição parcial esse valor irá ser menor, pois terá os custos da transportadora interna integralmente mais os custos da transportadora terceirizada.

Para facilitar a análise de viabilidade pode-se ainda calcular o valor de ganho por quilo do produto comercializado, neste mês foram carregados $3.000 .000 \mathrm{~kg}$ do produto, este valor é apresentado na Tabela 4.

Tabela 4. Valor ganho por quilo de produto

\begin{tabular}{ccc}
\hline Ganho & Quantidade transportada & Ganho/kg \\
\hline $\mathrm{R} \$$ & $\mathrm{~kg}$ & $\mathrm{R} \$$ \\
$14.989,46$ & 3.000 .000 & 0,005 \\
\hline
\end{tabular}

Na tabela 4 verifica-se que o ganho por kg de produto transportado não chega a um centavo, este é um valor muito importante para análise de viabilidade, ele representa o quanto seria possível repassar para o cliente com esta nova operação, um desconto a ser concedido, sem que gere uma diminuição na margem de lucro da empresa.

Analisando os valores de ganho financeiro o projeto é viável, mas não aceitável, pois mesmo com a substituição de $100 \%$ da transportadora terceirizada o valor por $\mathrm{kg}$ de produto transportado agregado é muito pequeno e tem as desvantagens desta operação que é deixar de contar com a teia de conhecimento desenvolvida pela transportadora nos vários anos de experiência no mercado, desenvolver uma operação que não é core business da empresa e no final o desconto que seria aplicado ao cliente, afim de ganhar ou manter as cotações seria de menos de $\mathrm{R} \$ 0,01$ (um centavo). Este valor teria que ser no mínimo 10 vezes maior para justificar o risco do novo empreendimento. E o ideal, ainda, seria que este ganho maior viesse de uma substituição parcial da transportadora terceirizada, pois não é saudável ter um único fornecedor. O relacionamento com a transportadora terceirizada mesmo que em uma escala pequena teria que ser mantido, pois poderão existir momentos de falta de caminhões, como acontece no auge na safra de grãos da região, um aumento de demanda momentâneo ou ainda cidades que tem pouco interesse por parte dos caminhões. Nestas situações ter a transportadora terceirizada como parceira seria de grande importância, no entanto uma operação em parceria diminuiria os lucros obtidos, 
pois o custo interno seria o mesmo para qualquer porcentagem de operação e teríamos que somar um custo proporcional a utilização da transportadora terceirizada.

\section{Conclusão}

Afim de verificar se a empresa contava com a opção mais rentável de transporte que este estudo foi realizado, chegando-se a conclusão que nas condições e considerações da análise realizada a alternativa de implantação de uma transportadora interna não é uma opção viável, pois apresenta pouca margem em relação a terceirização. A empresa permenecerá operando com a melhor alternativa.

Para um trabalho complementar uma analise de swat para transportadora interna poderia ser realizado, bem como uma analise financeira, envolvendo taxa interna de retorno - TIR.

\section{Referências}

Ballou, R.H. 2004.Gerenciamento da Cadeia de Suprimentos: Logística Empresarial. 5a ed. Porto Alegre: Artmed Editora S.A. 615 p.

Bowersox, D.J.; Closs, D.J. Logística Empresarial: O Processo de Integração da Cadeia de Suprimento. São Paulo: Editora Atlas S.A., 2001.

BRASIL. 2007. Lei n. 11.442, de 5 de janeiro de 2007. Dispõe sobre o transporte rodoviário de cargas por conta de terceiros e mediante remuneração e revoga a Lei no6.813, de 10 de julho de 1980. Disponível em: <http://www.planalto.gov.br/ccivil_03/_ato2007-2010/2007/lei/l11442.htm.> Acesso em: set, 2014.

Christopher, M. Logística e gerenciamento da cadeia de suprimentos. Criando rede que agregam valor. $2^{\underline{a}}$ ed. São Paulo: Thomson Learning, 2007.

Fleury, P.F.; Lavalle, C. 1995. O Estágio de Desenvolvimento da Organização

Logística em Grandes Empresas Brasileiras. Disponível em:

<http://professorricardo.tripod.com/Artigo_12.pdf.> Acesso em: set, 2014

Hoffmann, R.; Engler, J.J.C.; Serrano, O.; Thame, A.C.M.; Neves, E.M.

Administração da Empresa Agrícola, São Paulo: Pioneira, 1992. 325p.

Leiria, J.S.; Saratt, N.. Terceirização: uma alternativa de flexibilidade empresarial. 9 ed. São Paulo: Gente, 1996.

Portal tributário. 2014. O que é lucro presumido. Disponível em:

<http://www.portaltributario.com.br/artigos/oquee_lucropresumido.htm>

Acesso em: nov. 2014

Queiroz Ramos Soares de, C.A. Manual de Terceirização. 10. ed. São Paulo: STS, 1998. 
Secretária da fazenda, 2014. Definição de conhecimento de transporte eletrônico.

Disponível em:

$<$ http://www.cte.fazenda.gov.br/perguntasFrequentes.aspx?tipoConteudo=fYFul10Fiq $M=>$. Acesso em: nov. 2014.

Tabela de Alíquotas nas operações interestaduais. Disponível em: <http://www.boletimcontabil.com.br/tabelas/icms_orig.pdf.> Acesso em: set. 2014 


\section{Anexos}

ANEXO A. Alíquota de ICMS nas operações interestaduais

\section{DESTINO}

\begin{tabular}{|c|c|c|c|c|c|c|c|c|c|c|c|c|c|c|c|c|c|c|c|c|c|c|}
\hline & $\mathrm{AC}$ & \begin{tabular}{|l|} 
AL \\
\end{tabular} & $\mathbf{A M}$ & & $\mathrm{BA}$ & & & & MA & & & \begin{tabular}{l|l}
$\mathbf{I G}$ & $\mathbf{P}$ \\
\end{tabular} & \begin{tabular}{|l|l|}
$\mathrm{A}$ & $\mathrm{PB}$ \\
\end{tabular} & & \begin{tabular}{l|l}
$P E$ & $P$ \\
\end{tabular} & \begin{tabular}{l|l|}
$I$ & $\mathrm{RN}$ \\
\end{tabular} & \begin{tabular}{|l|l|} 
RS \\
\end{tabular} & \begin{tabular}{|l|l|l|l|}
$\mathrm{J}$ & $\mathrm{R}$ \\
\end{tabular} & \begin{tabular}{l|l}
$R R$ & $S$ \\
\end{tabular} & \begin{tabular}{l|l}
$\mathrm{SC}$ & $\mathrm{SF}$
\end{tabular} & \begin{tabular}{|l|l|}
$\mathrm{P}$ & $\mathrm{SE}$ \\
\end{tabular} & To \\
\hline $\mathrm{AC}$ & & 12 & 12 & \begin{tabular}{l|l}
12 & 1 \\
\end{tabular} & 12 & \begin{tabular}{l|l}
12 & 1
\end{tabular} & \begin{tabular}{l|l}
2.2 & 12 \\
\end{tabular} & 12 & 12 & 12 & 12 & \begin{tabular}{l|l}
12 & 1 \\
\end{tabular} & \begin{tabular}{l|l}
2 & 12 \\
\end{tabular} & & \begin{tabular}{l|l}
12 & 1
\end{tabular} & \begin{tabular}{l|l}
12 & 12
\end{tabular} & & \begin{tabular}{|l|l|}
2 & 12
\end{tabular} & & & \begin{tabular}{l|l|}
2 & 12 \\
\end{tabular} & 12 \\
\hline $\mathrm{AL}$ & 12 & & 12 & \begin{tabular}{l|l|l}
12 & \\
\end{tabular} & 12 & \begin{tabular}{l|l}
12 & 1 \\
\end{tabular} & \begin{tabular}{l|l|}
2.2 & 12 \\
\end{tabular} & 12 & 12 & 12 & 12 & \begin{tabular}{l|l}
12 & 1 \\
\end{tabular} & \begin{tabular}{|l|l|}
2 & 12
\end{tabular} & 12 & \begin{tabular}{l|l}
12 & 1 \\
\end{tabular} & \begin{tabular}{l|l}
12 & 12
\end{tabular} & \begin{tabular}{|l|l|l|l|}
12 & 12 \\
\end{tabular} & \begin{tabular}{|l|l|}
2 & 12
\end{tabular} & 12 & \begin{tabular}{l|l}
12 & 1 \\
\end{tabular} & \begin{tabular}{l|l|}
2 & 12 \\
\end{tabular} & 12 \\
\hline AM & 12 & 12 & & \begin{tabular}{l|l}
12 & 1 \\
\end{tabular} & 12 & \begin{tabular}{l|l}
12 & 1 \\
\end{tabular} & \begin{tabular}{l|l}
2 & 12 \\
\end{tabular} & 12 & 12 & 12 & $\begin{array}{ll}12 \\
\end{array}$ & \begin{tabular}{l|l}
12 & 1 \\
\end{tabular} & \begin{tabular}{|l|l|}
2 & 12 \\
\end{tabular} & 12 & \begin{tabular}{l|l}
12 & 1 \\
\end{tabular} & \begin{tabular}{l|l}
12 & 12
\end{tabular} & & \begin{tabular}{|l|l|}
2 & 12 \\
\end{tabular} & 12 & & 12 & 12 \\
\hline AP & 12 & 12 & 12 & & 12 & \begin{tabular}{l|l}
12 & 1 \\
\end{tabular} & \begin{tabular}{l|l|}
2.2 & 12 \\
\end{tabular} & 12 & 12 & 12 & 12 & \begin{tabular}{l|l}
12 & 1 \\
\end{tabular} & \begin{tabular}{l|l|}
2 & 12 \\
\end{tabular} & 12 & \begin{tabular}{l|l}
12 & 1 \\
\end{tabular} & \begin{tabular}{l|l}
12 & 12
\end{tabular} & \begin{tabular}{l|l}
12 & 12 \\
\end{tabular} & \begin{tabular}{|l|l|}
2 & 12
\end{tabular} & 12 & \begin{tabular}{l|l}
12 & 1 \\
\end{tabular} & \begin{tabular}{l|l|}
2 & 12 \\
\end{tabular} & 12 \\
\hline $\mathrm{BA}$ & 12 & 12 & 12 & 12 & & \begin{tabular}{l|l}
12 & 1 \\
\end{tabular} & \begin{tabular}{l|l}
2 & 12 \\
\end{tabular} & 12 & 12 & 12 & $\begin{array}{ll}12 \\
\end{array}$ & \begin{tabular}{l|l}
12 & 1 \\
\end{tabular} & \begin{tabular}{|l|l|}
2 & 12 \\
\end{tabular} & 12 & \begin{tabular}{l|l}
12 & 1 \\
\end{tabular} & \begin{tabular}{l|l}
12 & 12
\end{tabular} & \begin{tabular}{l|l}
12 & 12 \\
\end{tabular} & \begin{tabular}{|l|l|}
2 & 12 \\
\end{tabular} & 12 & & 12 & 12 \\
\hline$C E$ & 12 & 12 & 12 & 12 & 12 & & \begin{tabular}{l|l}
2 & 12 \\
\end{tabular} & 12 & 12 & 12 & $\begin{array}{ll}12 \\
\end{array}$ & \begin{tabular}{l|l}
12 & 1 \\
\end{tabular} & \begin{tabular}{|l|l|}
2 & 12 \\
\end{tabular} & 12 & 12 & 12 & \begin{tabular}{l|l}
12 & 12 \\
\end{tabular} & \begin{tabular}{|l|l|}
2 & 12 \\
\end{tabular} & 12 & \begin{tabular}{l|l}
12 & 1 \\
\end{tabular} & \begin{tabular}{|l|l|}
2 & 12 \\
\end{tabular} & 12 \\
\hline DF & 12 & 12 & 12 & 12 & 12 & 12 & 12 & 12 & 12 & 12 & 12 & \begin{tabular}{l|l}
12 & 1 \\
\end{tabular} & \begin{tabular}{|l|l|}
2 & 12 \\
\end{tabular} & 12 & \begin{tabular}{l|l}
12 & 1 \\
\end{tabular} & 12 & \begin{tabular}{|l|l}
12 & 12 \\
\end{tabular} & \begin{tabular}{|l|l|}
2 & 12 \\
\end{tabular} & 12 & 12 & 12 & 12 \\
\hline ES & 12 & 12 & 12 & 12 & 12 & \begin{tabular}{l|l}
12 & 1 \\
\end{tabular} & 2 & 12 & 12 & 12 & $\begin{array}{ll}12 \\
\end{array}$ & \begin{tabular}{l|l}
12 & 1 \\
\end{tabular} & \begin{tabular}{|l|l|}
2 & 12 \\
\end{tabular} & 12 & 12 & 12 & \begin{tabular}{|l|l}
12 & 12 \\
\end{tabular} & \begin{tabular}{|l|l|}
2 & 12 \\
\end{tabular} & 12 & & 12 & 12 \\
\hline GO & 12 & 12 & 12 & 12 & 12 & \begin{tabular}{l|l}
12 & 1 \\
\end{tabular} & \begin{tabular}{l|l}
2 & 12 \\
\end{tabular} & & 12 & 12 & 12 & \begin{tabular}{l|l}
12 & 1 \\
\end{tabular} & \begin{tabular}{|l|l|}
12 & 12 \\
\end{tabular} & 12 & \begin{tabular}{l|l}
12 & 1 \\
\end{tabular} & $\begin{array}{ll}12 & 12\end{array}$ & \begin{tabular}{|l|l}
12 & 12 \\
\end{tabular} & \begin{tabular}{|l|l|}
2 & 12 \\
\end{tabular} & 12 & 12 & \begin{tabular}{|l|l|}
2 & 12 \\
\end{tabular} & 12 \\
\hline MA & 12 & 12 & 12 & 12 & 12 & \begin{tabular}{l|l}
12 & 1 \\
\end{tabular} & \begin{tabular}{l|l}
2 & 12 \\
\end{tabular} & 12 & & 12 & 12 & \begin{tabular}{l|l}
12 & 1 \\
\end{tabular} & & 12 & & $\begin{array}{ll}12 & 12\end{array}$ & \begin{tabular}{|l|l}
12 & 12 \\
\end{tabular} & \begin{tabular}{|l|l|}
2 & 12 \\
\end{tabular} & 12 & & \begin{tabular}{|l|l|}
2 & 12 \\
\end{tabular} & 12 \\
\hline MT & 12 & 12 & 12 & 12 & 12 & \begin{tabular}{l|l}
12 & 1 \\
\end{tabular} & 12 & 12 & 12 & & 12 & \begin{tabular}{l|l}
12 & 1 \\
\end{tabular} & \begin{tabular}{|l|l|}
2 & 12 \\
\end{tabular} & 12 & \begin{tabular}{l|l}
12 & 1 \\
\end{tabular} & \begin{tabular}{l|l}
12 & 12
\end{tabular} & \begin{tabular}{|l|l}
12 & 12 \\
\end{tabular} & \begin{tabular}{|l|l|}
2 & 12 \\
\end{tabular} & 12 & & \begin{tabular}{|l|l|}
2 & 12 \\
\end{tabular} & 12 \\
\hline MS & 12 & 12 & 12 & 12 & 12 & \begin{tabular}{l|l}
12 & 1 \\
\end{tabular} & \begin{tabular}{|l|l|l|}
2 & 12 \\
\end{tabular} & 12 & 12 & 12 & & \begin{tabular}{l|l}
12 & 1 \\
\end{tabular} & \begin{tabular}{|l|l|}
12 & 12 \\
\end{tabular} & 12 & $\begin{array}{ll}12 & 1\end{array}$ & \begin{tabular}{l|l}
12 & 12
\end{tabular} & \begin{tabular}{|l|l}
12 & 12 \\
\end{tabular} & \begin{tabular}{|l|l|}
2 & 12 \\
\end{tabular} & 12 & & \begin{tabular}{|l|l|}
2 & 12 \\
\end{tabular} & 12 \\
\hline MG & 7 & 7 & 7 & 7 & 7 & 7 & \begin{tabular}{l|l}
7 & 7 \\
\end{tabular} & 7 & 7 & 7 & 7 & & \begin{tabular}{|l|l|}
7 & 7 \\
\end{tabular} & 12 & 7 & 7 & \begin{tabular}{|l|l}
12 & 12 \\
\end{tabular} & \begin{tabular}{|l|l|}
2 & 7 \\
\end{tabular} & 7 & & 12 & 7 \\
\hline $\mathrm{PA}$ & 12 & 12 & 12 & 12 & 12 & \begin{tabular}{l|l}
12 & 1 \\
\end{tabular} & \begin{tabular}{l|l}
12 & 12 \\
\end{tabular} & 12 & 12 & 12 & 12 & 12 & 12 & 12 & \begin{tabular}{l|l}
12 & 1 \\
\end{tabular} & 12 & \begin{tabular}{|l|l}
12 & 12 \\
\end{tabular} & \begin{tabular}{|l|l|}
2 & 12 \\
\end{tabular} & 12 & & \begin{tabular}{|l|l|}
12 & 12 \\
\end{tabular} & 12 \\
\hline PB & 12 & 12 & 12 & 12 & 12 & 12 & \begin{tabular}{l|l}
12 & 12 \\
\end{tabular} & 12 & 12 & 12 & 12 & \begin{tabular}{l|l}
12 & 1 \\
\end{tabular} & 12 & 12 & \begin{tabular}{l|l}
12 & 1
\end{tabular} & \begin{tabular}{l|l}
12 & 12
\end{tabular} & \begin{tabular}{|l|l|}
12 & 12 \\
\end{tabular} & \begin{tabular}{|l|l|}
2 & 12 \\
\end{tabular} & 12 & \begin{tabular}{l|l}
12 & 1
\end{tabular} & \begin{tabular}{|l|l|}
12 & 12 \\
\end{tabular} & 12 \\
\hline $\begin{array}{l}\mathrm{PR} \\
\end{array}$ & 7 & 7 & 7 & 7 & 7 & 7 & $\begin{array}{l}77 \\
\end{array}$ & 7 & 7 & 7 & 7 & 127 & 77 & & 7 & $\begin{array}{ll}77 \\
\end{array}$ & 1212 & 27 & 7 & 121 & \begin{tabular}{l|l|}
12 & 7 \\
\end{tabular} & 7 \\
\hline $\mathrm{PE}$ & 12 & 12 & 12 & 12 & 12 & 121 & \begin{tabular}{l|l}
12 & 12 \\
\end{tabular} & 12 & 12 & 12 & 12 & \begin{tabular}{l|l}
12 & 1 \\
\end{tabular} & \begin{tabular}{l|l|}
12 & 12 \\
\end{tabular} & 12 & & 12 & \begin{tabular}{l|l}
12 & 12 \\
\end{tabular} & \begin{tabular}{|l|l|}
2 & 12 \\
\end{tabular} & 12 & 12 & \begin{tabular}{l|l}
12 & 12 \\
\end{tabular} & 12 \\
\hline PI & 12 & 12 & 12 & 12 & 12 & 121 & \begin{tabular}{l|l}
12 & 12 \\
\end{tabular} & 12 & 12 & 12 & 12 & \begin{tabular}{l|l}
12 & 1 \\
\end{tabular} & \begin{tabular}{|l|l|}
12 & 12 \\
\end{tabular} & 12 & 12 & 12 & \begin{tabular}{|l|l}
12 & 12 \\
\end{tabular} & \begin{tabular}{|l|l|}
2 & 12 \\
\end{tabular} & 12 & & \begin{tabular}{l|l}
12 & 12 \\
\end{tabular} & 12 \\
\hline $\mathrm{RN}$ & 12 & 12 & 12 & 12 & 12 & 121 & 12 & 12 & 12 & 12 & 12 & 12 & \begin{tabular}{|l|l|}
12 & 12 \\
\end{tabular} & 12 & 12 . & 12 & \begin{tabular}{|l|l}
12 & 12 \\
\end{tabular} & \begin{tabular}{|l|l|}
2 & 12 \\
\end{tabular} & 12 & 12 & \begin{tabular}{l|l}
12 & 12 \\
\end{tabular} & 12 \\
\hline RS & 7 & 7 & 7 & 7 & 7 & 7 & \begin{tabular}{l|l}
7 & 7
\end{tabular} & 7 & 7 & 7 & 7 & 12 & \begin{tabular}{l|l}
7 & 7
\end{tabular} & 12 & 7 & 7 & 12 & \begin{tabular}{|l|l|}
2 & 7 \\
\end{tabular} & 7 & & \begin{tabular}{l|l}
12 & 7
\end{tabular} & \\
\hline RJ & 7 & 7 & 7 & 7 & 7 & 7 & 7 & 7 & 7 & 7 & 7 & 12 & 7 & 12 & 7 & 7 & 12 & 7 & 7 & \begin{tabular}{l|l}
12 & 1
\end{tabular} & 12 & 7 \\
\hline RO & 12 & 12 & 12 & 12 & 12 & 12 & 12 & 12 & 12 & 12 & 12 & 12 & 12 & 12 & 12 & 12 & 12 & & 12 & & 12 & 12 \\
\hline RR & 12 & 12 & 12 & 12 & 12 & 121 & \begin{tabular}{l|l}
12 & 12 \\
\end{tabular} & 12 & 12 & 12 & 12 & 12 & \begin{tabular}{|l|l}
12 & 12 \\
\end{tabular} & 12 & 12 & \begin{tabular}{l|l}
12 & 12 \\
\end{tabular} & \begin{tabular}{|l|l}
12 & 12 \\
\end{tabular} & \begin{tabular}{|l|l|}
2 & 12 \\
\end{tabular} & & \begin{tabular}{l|l}
12 & 1
\end{tabular} & \begin{tabular}{l|l}
12 & 12
\end{tabular} & 12 \\
\hline SC & 7 & 7 & 7 & 7 & 7 & 7 & 7 & 7 & \begin{tabular}{|l|}
7 \\
\end{tabular} & 7 & 7 & 12 & \begin{tabular}{|l|l}
7 & 7
\end{tabular} & 12 & 7 & \begin{tabular}{|l|l}
7 & 7
\end{tabular} & \begin{tabular}{|l|l|}
12 & 12 \\
\end{tabular} & \begin{tabular}{|l|l}
2 & 7
\end{tabular} & 7 & & \begin{tabular}{l|l}
12 & 7
\end{tabular} & \\
\hline SP & 7 & 7 & 7 & 7 & 7 & 7 & 7 & 7 & 7 & 7 & 7 & 12 & 7 & 12 & 7 & 7 & \begin{tabular}{|l|l|}
12 & 12 \\
\end{tabular} & 7 & 7 & 12 & 7 & 7 \\
\hline SE & 12 & 12 & 12 & 12 & 12 & 12 & 12 & & 12 & 12 & & & & & & 12 & \begin{tabular}{|l|l}
12 & 12 \\
\end{tabular} & 12 & & & & \\
\hline To & 12 & 12 & & & & 121 & & & & & & & & & & & & & & & & \\
\hline
\end{tabular}

Referência: Tabela de Alíquotas nas operações interestaduais, 2014 
Anexo B. Dados amostrais utilizados para cálculos da margem obtida pela transportadora

\begin{tabular}{|c|c|c|c|c|c|c|c|c|c|}
\hline VPM & VPT & RECEITA 1 & $\begin{array}{c}\text { PIS/ } \\
\text { CONFNS }\end{array}$ & ICMS & $\begin{array}{l}\text { VALOR DE } \\
\text { IMPOSTOS }\end{array}$ & $\begin{array}{c}\text { VALOR DA } \\
\text { MERCADORIA }\end{array}$ & SEGURO & RECEITA FNAL & $\begin{array}{c}\text { MARGEM } \\
\text { OBTIDA }\end{array}$ \\
\hline $\mathbf{R} \$$ & $\mathbf{R} \$$ & $\mathbf{R} \$$ & & & $\mathbf{R} \$$ & $\mathbf{R} \$$ & $\mathbf{R} \$$ & $\mathbf{R} \$$ & $\%$ \\
\hline $1.400,00$ & $1.920,00$ & 520,00 & $3,65 \%$ & $0,00 \%$ & 70,08 & $35.801,60$ & 143,21 & 306,71 & 15,97 \\
\hline $1.050,00$ & $1.470,00$ & 420,00 & $3,65 \%$ & $7,00 \%$ & 156,56 & $16.130,44$ & 64,52 & 198,92 & 13,53 \\
\hline $3.000,00$ & $4.350,00$ & $1.350,00$ & $3,65 \%$ & $12,00 \%$ & 680,78 & $35.400,00$ & 141,60 & 527,63 & 12,13 \\
\hline $2.850,00$ & $4.050,00$ & $1.200,00$ & $3,65 \%$ & $12,00 \%$ & 633,83 & $45.000,00$ & 180,00 & 386,18 & 9,54 \\
\hline $1.400,00$ & $1.890,00$ & 490,00 & $3,65 \%$ & $12,00 \%$ & 295,79 & $18.630,00$ & 74,52 & 119,70 & 6,33 \\
\hline $1.500,00$ & $2.025,00$ & 525,00 & $3,65 \%$ & $12,00 \%$ & 316,91 & $16.605,00$ & 66,42 & 141,67 & 7,00 \\
\hline $1.300,00$ & $1.890,00$ & 590,00 & $3,65 \%$ & $12,00 \%$ & 295,79 & $19.170,00$ & 76,68 & 217,54 & 11,51 \\
\hline $2.405,00$ & $3.330,00$ & 925,00 & $3,65 \%$ & $12,00 \%$ & 521,15 & $42.550,00$ & 170,20 & 233,66 & 7,02 \\
\hline $2.200,00$ & $2.990,00$ & 790,00 & $3,65 \%$ & $12,00 \%$ & 467,94 & $31.500,00$ & 126,00 & 196,07 & 6,56 \\
\hline $1.100,00$ & $1.560,00$ & 460,00 & $3,65 \%$ & $0,00 \%$ & 56,94 & $26.000,00$ & 104,00 & 299,06 & 19,17 \\
\hline 800,00 & 945,00 & 145,00 & $3,65 \%$ & $7,00 \%$ & 100,64 & $15.120,00$ & 60,48 & $-16,12$ & $-1,71$ \\
\hline $2.500,00$ & $3.150,00$ & 650,00 & $3,65 \%$ & $12,00 \%$ & 492,98 & $30.975,00$ & 123,90 & 33,13 & 1,05 \\
\hline $2.400,00$ & $3.536,00$ & $1.136,00$ & $3,65 \%$ & $12,00 \%$ & 553,38 & $37.280,00$ & 149,12 & 433,50 & 12,26 \\
\hline $2.600,00$ & $3.600,00$ & $1.000,00$ & $3,65 \%$ & $12,00 \%$ & 563,40 & $41.700,00$ & 166,80 & 269,80 & 7,49 \\
\hline $1.000,00$ & $1.377,00$ & 377,00 & $3,65 \%$ & $12,00 \%$ & 215,50 & $18.630,00$ & 74,52 & 86,98 & 6,32 \\
\hline $1.400,00$ & $1.600,00$ & 200,00 & $3,65 \%$ & $12,00 \%$ & 250,40 & $15.727,50$ & 62,91 & $-113,31$ & $-7,08$ \\
\hline 800,00 & $1.080,00$ & 280,00 & $3,65 \%$ & $0,00 \%$ & 39,42 & $15.930,00$ & 63,72 & 176,86 & 16,38 \\
\hline $2.900,00$ & $3.990,00$ & $1.090,00$ & $3,65 \%$ & $7,00 \%$ & 424,94 & $42.300,00$ & 169,20 & 495,87 & 12,43 \\
\hline $1.000,00$ & $1.620,00$ & 620,00 & $3,65 \%$ & $12,00 \%$ & 253,53 & $16.065,00$ & 64,26 & 302,21 & 18,65 \\
\hline $2.200,00$ & $3.000,00$ & 800,00 & $3,65 \%$ & $12,00 \%$ & 469,50 & $29.700,00$ & 118,80 & 211,70 & 7,06 \\
\hline $1.100,00$ & $1.600,00$ & 500,00 & $3,65 \%$ & $12,00 \%$ & 250,40 & $13.050,00$ & 52,20 & 197,40 & 12,34 \\
\hline $3.000,00$ & $4.350,00$ & $1.350,00$ & $3,65 \%$ & $12,00 \%$ & 680,78 & $35.400,00$ & 141,60 & 527,63 & 12,13 \\
\hline $3.300,00$ & $4.590,00$ & $1.290,00$ & $3,65 \%$ & $7,00 \%$ & 488,84 & $19.440,00$ & 77,76 & 723,41 & 15,76 \\
\hline $1.100,00$ & $1.404,00$ & 304,00 & $3,65 \%$ & $7,00 \%$ & 149,53 & $16.537,50$ & 66,15 & 88,32 & 6,29 \\
\hline $1.400,00$ & $1.890,00$ & 490,00 & $3,65 \%$ & $12,00 \%$ & 295,79 & $18.630,00$ & 74,52 & 119,70 & 6,33 \\
\hline $3.000,00$ & $4.800,00$ & $1.800,00$ & $3,65 \%$ & $12,00 \%$ & 751,20 & $36.000,00$ & 144,00 & 904,80 & 18,85 \\
\hline $2.400,00$ & $3.600,00$ & $1.200,00$ & $3,65 \%$ & $12,00 \%$ & 563,40 & $36.000,00$ & 144,00 & 492,60 & 13,68 \\
\hline $1.600,00$ & $1.890,00$ & 290,00 & $3,65 \%$ & $7,00 \%$ & 201,29 & $16.200,00$ & 64,80 & 23,91 & 1,27 \\
\hline $1.000,00$ & $1.485,00$ & 485,00 & $3,65 \%$ & $0,00 \%$ & 54,20 & $18.285,00$ & 73,14 & 357,66 & 24,08 \\
\hline $1.200,00$ & $1.957,50$ & 757,50 & $3,65 \%$ & $12,00 \%$ & 306,35 & $14.175,00$ & 56,70 & 394,45 & 20,15 \\
\hline $2.500,00$ & $3.600,00$ & $1.100,00$ & $3,65 \%$ & $12,00 \%$ & 563,40 & $39.300,00$ & 157,20 & 379,40 & 10,54 \\
\hline $2.200,00$ & $3.000,00$ & 800,00 & $3,65 \%$ & $12,00 \%$ & 469,50 & $34.500,00$ & 138,00 & 192,50 & 6,42 \\
\hline $3.000,00$ & $4.160,00$ & $1.160,00$ & $3,65 \%$ & $12,00 \%$ & 651,04 & $39.190,00$ & 156,76 & 352,20 & 8,47 \\
\hline 800,00 & $1.255,50$ & 455,50 & $3,65 \%$ & $0,00 \%$ & 45,83 & $15.930,00$ & 63,72 & 345,95 & 27,56 \\
\hline 800,00 & 945,00 & 145,00 & $3,65 \%$ & $7,00 \%$ & 100,64 & $15.120,00$ & 60,48 & $-16,12$ & $-1,71$ \\
\hline $2.400,00$ & $3.600,00$ & $1.200,00$ & $3,65 \%$ & $12,00 \%$ & 563,40 & $41.700,00$ & 166,80 & 469,80 & 13,05 \\
\hline $2.200,00$ & $2.990,00$ & 790,00 & $3,65 \%$ & $12,00 \%$ & 467,94 & $31.500,00$ & 126,00 & 196,07 & 6,56 \\
\hline $1.300,00$ & $1.822,50$ & 522,50 & $3,65 \%$ & $12,00 \%$ & 285,22 & $17.955,00$ & 71,82 & 165,46 & 9,08 \\
\hline $2.600,00$ & $3.150,00$ & 550,00 & $3,65 \%$ & $12,00 \%$ & 492,98 & $34.650,00$ & 138,60 & $-81,57$ & $-2,59$ \\
\hline $2.100,00$ & $3.536,00$ & $1.436,00$ & $3,65 \%$ & $12,00 \%$ & 553,38 & $37.280,00$ & 149,12 & 733,50 & 20,74 \\
\hline $1.000,00$ & $1.282,50$ & 282,50 & $3,65 \%$ & $0,00 \%$ & 46,81 & $15.255,00$ & 61,02 & 174,67 & 13,62 \\
\hline $1.100,00$ & $1.485,00$ & 385,00 & $3,65 \%$ & $12,00 \%$ & 232,40 & $17.415,00$ & 69,66 & 82,94 & 5,59 \\
\hline $76.905,00$ & $107.766,00$ & $30.861,00$ & & & $15.073,72$ & $1.113 .727,04$ & $4.454,91$ & $11.332,37$ & 10,52 \\
\hline
\end{tabular}




\section{Anexo C. Modelo de Conhecimento de Transporte Eletrônico}
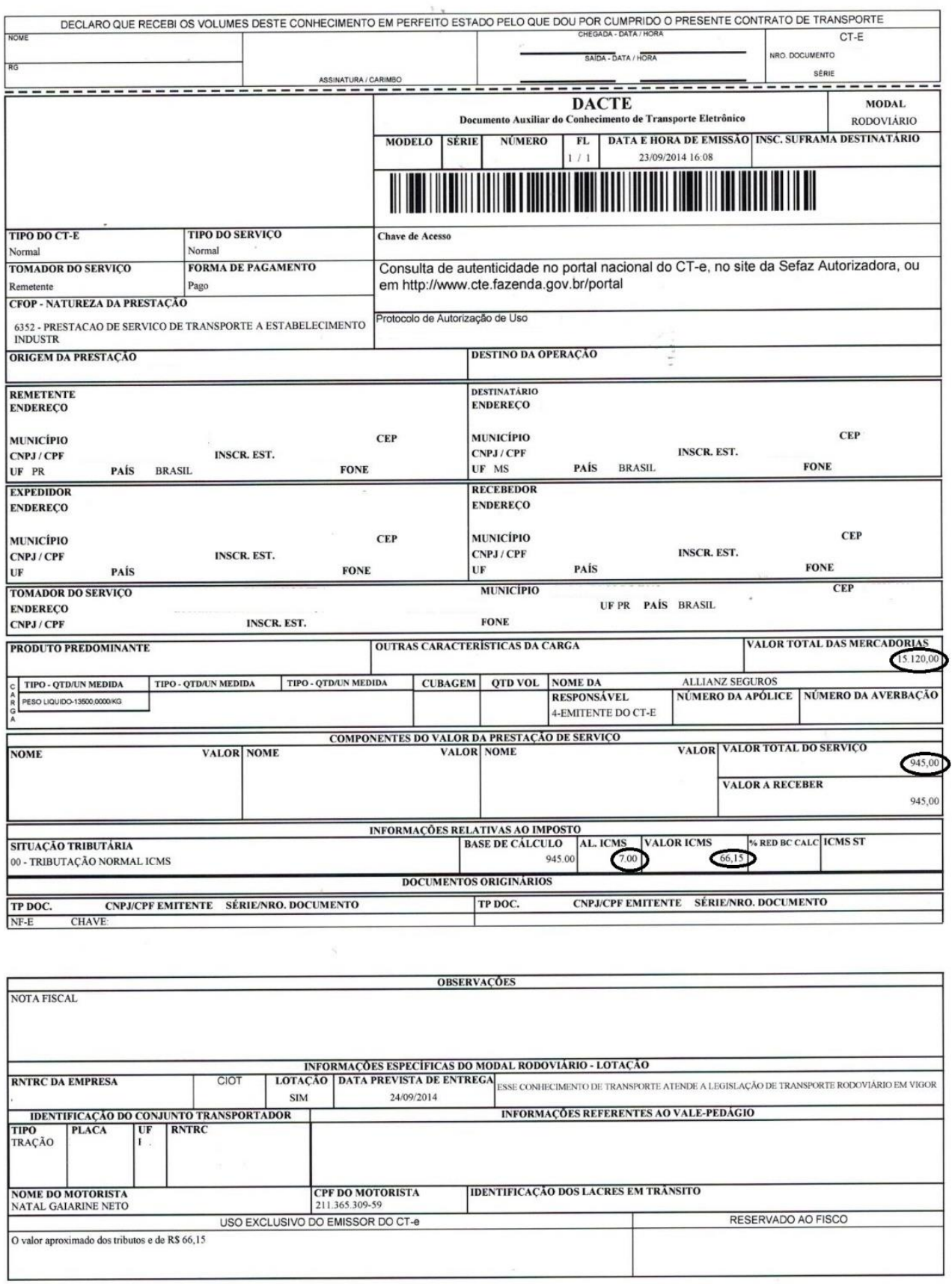

Referência: Modelo utilizado pela transportadora terceirizada. 